\title{
Epidemiological Survey on Porcine Cysticercosis in Nay Pyi Taw Area, Myanmar
}

\author{
Tin Aye Khaing, ${ }^{1,2}$ Saw Bawm, ${ }^{2}$ Soe Soe Wai, ${ }^{2}$ Ye Htut, ${ }^{1}$ and Lat Lat Htun ${ }^{2}$ \\ ${ }^{1}$ Department of Veterinary and Slaughterhouses, Nay Pyi Taw Development Committee, Nay Pyi Taw, Myanmar \\ ${ }^{2}$ Department of Pharmacology and Parasitology, University of Veterinary Science, Nay Pyi Taw 15013, Myanmar \\ Correspondence should be addressed to Saw Bawm; bestshadow@gmail.com
}

Received 30 September 2014; Revised 6 January 2015; Accepted 16 February 2015

Academic Editor: Raul J. Bobes

Copyright (c) 2015 Tin Aye Khaing et al. This is an open access article distributed under the Creative Commons Attribution License, which permits unrestricted use, distribution, and reproduction in any medium, provided the original work is properly cited.

\begin{abstract}
Cross-sectional surveys were conducted to determine the prevalence and associated risk factors of Taenia solium cysticercosis in pigs within Nay Pyi Taw area, Myanmar. Meat inspection in three slaughterhouses, ELISA test, and questionnaire surveys were conducted in this study. Three hundred pigs were inspected in slaughterhouses and 364 pigs were randomly selected and examined from 203 households from three townships in Nay Pyi Taw area. The prevalence of porcine cysticercosis in meat inspection was $23.67 \%$ (71/300). Seroprevalence of T. solium cysticercosis in pigs in the study area was $15.93 \%(58 / 364)$. Significant associated risk factors with $T$. solium cysticercosis were gender $(\mathrm{OR}=3.0 ; 95 \% \mathrm{CI}=1.7-5.4)$, increased age $(\mathrm{OR}=2.3 ; 95 \% \mathrm{CI}=1.2-4.2)$, husbandry system $(\mathrm{OR}=5.1 ; 95 \% \mathrm{CI}=2.4-11.2)$, feed type $(\mathrm{OR}=16.9 ; 95 \% \mathrm{CI}=2.3-124.3)$, not using anthelmintics in pigs $(\mathrm{OR}=11.9 ; 95 \% \mathrm{CI}=5.0-28.5)$, not using anthelmintics in owner $(\mathrm{OR}=2.5 ; 95 \% \mathrm{CI}=1.4-4.4)$, no hand-washing before feeding $(\mathrm{OR}=31.5 ; 95 \% \mathrm{CI}=4.3-230.9)$, and pork consumption of owner $(\mathrm{OR}=37.4 ; 95 \% \mathrm{CI}=9.0-156.1)$ in the study area. This is the first report of porcine cysticercosis in Myanmar.
\end{abstract}

\section{Introduction}

Human neurocysticercosis (NCC) is caused by larval stage of zoonotic tapeworm Taenia solium (pork tapeworm) which remains a major public health problem in developing and some developed countries [1]. The World Health Organization estimates that eight people per 1000 worldwide have NCC [2]. This leads to epilepsy, madness, blindness, and death [3]. NCC can also occur in individuals who do not raise pigs or consume pork. Porcine cysticercosis is the cause of human taeniasis and neurocysticercosis is a consequence of taeniasis. Based on the available information, a very conservative and rough economic estimate indicates that the annual losses due to porcine cysticercosis in 10 west and central African countries amount to about 25 million Euros [4]. Ito et al. [5] also stated that, in China, the amount of pork discarded in the whole country due to cysticercosis annually has been estimated as $200,000,000 \mathrm{~kg}$ with a value of more than US $\$ 120,000,000$. Dorny et al. [6] stated that notably data on Myanmar are lacking, although there are several reports of porcine cysticercosis based on meat inspection in the abattoirs in neighboring countries, 9.3\% in India [7], 32.5\% in Nepal [8], 5.4\% in China [9], 0.02-2.63\% in Indonesia [10], and 0.04 to $0.9 \%$ in Vietnam [6].

Although most of Myanmar culinary habits are based on thorough cooking, new food style such as barbecue and dishes based on raw or undercooked pork or pork product becomes popular among customers. Moreover, small-scale pig husbandry has become one of the major sources of income in Myanmar farmers. So it may be high risk of getting food-borne zoonotic diseases according to the new food style and traditional husbandry method. Due to lacking of information on porcine cysticercosis in Myanmar up to now, it is important to investigate the prevalence and associated risk factors.

Nay Pyi Taw area, the capital of Myanmar, has big population of pigs (about 200,000 pigs) [11] to support the demand of pork consumption in this area. Most of 
the pig farmers are smallholders and most of pig husbandry systems are free ranging or semi-intensive with lack of proper sanitation.

One of the main obstacles to control the T. solium infections is the lack of adequate epidemiological data on cysticercosis/taeniasis. Therefore, the objectives of this community-based study were to investigate the prevalence of porcine cysticercosis and associated risk factors in pigs within study area. Moreover, findings of this study will assist to develop the control strategies of porcine cysticercosis for the public health aspect.

\section{Materials and Methods}

2.1. Study Design, Study Area, and Sample Size. The crosssectional studies were conducted from January to March and June to July 2014, to investigate the prevalence of Taenia solium cysticercosis in slaughtered and farmed pigs within Pyinmana, Lewe, and Tatkon townships, Nay Pyi Taw area. It is located between latitude $19^{\circ} 45^{\prime} \mathrm{N}$ and longitude $96^{\circ} 6^{\prime} \mathrm{E}$ and with climate data; the altitude is $115 \mathrm{~m}$ above sea level, annual rainfall is $115 \mathrm{~mm}$, and annual temperature is $21.2-32.5^{\circ} \mathrm{C}$. The targeted population was 180,000 pigs in three townships [11] during the sampling period. The number of sampled pigs was calculated using the formula stated by Thrusfield [12]. An expected prevalence of $30 \%$ with a confidence level of $95 \%$ was used in this unit. In this study, 300 slaughtered pigs and 364 farmed pigs from the study area were examined although calculated samples were 298 and 323, respectively (Table 1). Blood collected from the jugular vein of farmed pig was conducted for the seroprevalence and a structured questionnaire with both closed and open-ended questions was administered to owners to obtain management practices in pig husbandry. Piglets younger than two months, pregnant sows, and nursing sows with litters less than two months old were excluded from this study to overcome the stress which causes adverse effect in animals.

2.2. Meat Inspection in Slaughterhouses. Meat inspection was carried out as described by Boa et al. [13] in the three slaughterhouses of these townships. There were 300 randomly selected pigs recruited and 9 different muscles (tongue, masseter, brain, shoulder, diaphragmatic, heart, skeletal, fore limb, and hind limb muscle) from each pig in meat inspection. Briefly, long and parallel incision into the masseter muscles on both sides of face in an upward direction was made. A deep longitudinal incision covering about 3/4 the thickness of the tongue and covering the whole length of the tongue was made to examine the cysts. After opening the pericardium, the heart was also visually examined for the presence of cysts. The heart was cut open and a deep (3/4 the thickness of septum) incision into the septum was made to expose any metacestodes. All the other muscles were viewed, palpated, incised by surgical blade, and visually examined.

2.3. Blood Collection and Antibody-ELISA Test for the Detection of IgG Antibody of T. solium Cysticerci. The pig was kept under restraint at standing position and blood samples
TABLE 1: Distribution of the number of samples in pigs within the three townships for blood collection.

\begin{tabular}{lccc}
\hline Number & Township & Pig population & Number of sampled pigs \\
\hline 1 & Pyinmana & 39,000 & 81 \\
2 & Lewe & 86,000 & 172 \\
3 & Tatkon & 55,000 & 111 \\
\hline & Total & 180,000 & 364 \\
\hline
\end{tabular}

were obtained from the external jugular vein by using sterile disposable syringes and put into vacutainers with clot activators. Those vacutainers were kept in cold boxes with ice and transported to Department of Pharmacology and Parasitology, University of Veterinary Science, Nay Pyi Taw, and allowed overnight at $4^{\circ} \mathrm{C}$ to clot. To obtain serum, the clotted blood was separated by centrifugation at $4000 \mathrm{rpm}$ for 10 minutes. The clear sera were transferred to $1.5 \mathrm{~mL}$ microvial tubes and stored in labeled wails and kept at $-20^{\circ} \mathrm{C}$ until analysis.

Detection of IgG antibody of $T$. solium cysticerci was carried out by using antibody-ELISA kit (NovaTec Immundiagnostica GMBH Co., Belgium) according to manufacturer's instruction. Briefly, all thawed samples were diluted as $1+100$ with IgG Sample Diluent (phosphate buffer) before assaying. The $100 \mu \mathrm{L}$ controls and diluted samples were dispensed into their respective wells and the foil was covered. After incubation for 1 hour at $37^{\circ} \mathrm{C}$ and the foil being removed, the contents of the wells were aspirated and washed three times with washing solution. And then, remaining fluid was carefully removed by tapping strips on tissue paper. The $100 \mu \mathrm{L}$ protein A conjugate (horseradish peroxidase) was dispensed into all wells except A1 and covered with foil and incubated for $30 \mathrm{~min}$ at room temperature. After washing three times, $100 \mu \mathrm{L}$ TMB $\left(3,3^{\prime}, 5,5^{\prime}\right.$-tetramethylbenzidine) substrate solution was dispensed into all wells and incubated for exactly $15 \mathrm{~min}$ at room temperature in the dark. The reaction was stopped by adding $100 \mu \mathrm{L}$ stop solution $(0.2 \mathrm{M}$ $\left.\mathrm{H}_{2} \mathrm{SO}_{4}\right)$. The absorbance was determined at $450 / 620 \mathrm{~nm}$ using an ELISA reader (Stat Fax). In each ELISA kit testing, there are two cut-off controls $\left(\mathrm{C}_{1}\right.$ and $\left.\mathrm{D}_{1}\right)$. The mean absorbance of these cut-off controls was used as cut-off value. Samples are considered "positive" if the absorbance value is higher than $10 \%$ over the cut-off and samples are considered "negative" if the absorbance value is lower than $10 \%$ below the cut-off. The sensitivity and specificity of these kits to diagnose swine cysticercosis are $93.8 \%$ and $>95 \%$, respectively.

2.4. Household Questionnaire. A questionnaire was developed and used to collect information on hypothesized risk factors and other related pieces of information from sampled pig owners. Households in each township were selected by using the snowballing technique from those farmers willing to participate in the study. It is a technique for developing a research sample where existing study subjects recruit future subjects from their acquaintances.

2.5. Statistical Analysis. The questionnaire interviewed data were analyzed for the relationship between the prevalence 
TABLE 2: Distribution and odds ratio of associated risk factors concerning porcine cysticercosis in Nay Pyi Taw area.

\begin{tabular}{|c|c|c|c|c|c|c|}
\hline Factor & Level & $n$ & Positive case & Negative case & Odds ratio & $P$ value \\
\hline \multirow{2}{*}{ Gender } & Male & 221 & 22 & 199 & \multirow{2}{*}{$3.043(1.704-5.436)$} & \multirow{2}{*}{$0.000^{*}$} \\
\hline & Female & 143 & 36 & 107 & & \\
\hline \multirow{2}{*}{ Age } & $<6$ month & 291 & 39 & 252 & \multirow{2}{*}{$2.274(1.220-4.235)$} & \multirow{2}{*}{$0.012^{*}$} \\
\hline & $\geq 6$ month & 73 & 19 & 54 & & \\
\hline \multirow{2}{*}{ Husbandry system } & Intensive & 146 & 8 & 138 & \multirow{2}{*}{$5.134(2.354-11.195)$} & \multirow{2}{*}{$0.000^{*}$} \\
\hline & Semi-intensive & 218 & 50 & 168 & & \\
\hline \multirow{2}{*}{ Feed type } & Only commercial feed & 71 & 1 & 70 & \multirow{2}{*}{$16.907(2.300-124.302)$} & \multirow{2}{*}{$0.000^{*}$} \\
\hline & Both with kitchen waste & 293 & 57 & 236 & & \\
\hline \multirow{2}{*}{ Use of anthelmintic in pigs } & Yes & 183 & 6 & 177 & \multirow{2}{*}{$11.891(4.957-28.526)$} & \multirow{2}{*}{$0.000^{*}$} \\
\hline & No & 181 & 52 & 129 & & \\
\hline \multirow{2}{*}{ Use of anthelmintic in owner } & Yes & 242 & 28 & 214 & \multirow{2}{*}{$2.492(1.409-4.407)$} & \multirow{2}{*}{$0.002^{*}$} \\
\hline & No & 122 & 30 & 92 & & \\
\hline \multirow{2}{*}{ Hand-washing before feeding } & Yes & 110 & 1 & 119 & \multirow{2}{*}{31.538 (4.307-230.92) } & \multirow{2}{*}{$0.000^{*}$} \\
\hline & No & 254 & 57 & 197 & & \\
\hline \multirow{2}{*}{ Pork consumption of owner } & No & 177 & 2 & 175 & \multirow{2}{*}{37.405 (8.965-156.068) } & \multirow{2}{*}{$0.000^{*}$} \\
\hline & Yes & 187 & 56 & 131 & & \\
\hline
\end{tabular}

${ }^{*}$ Significant association at 0.05 level.

of T. solium cysticercosis and hypothesized risk variables such as age, gender of pigs, husbandry system, feed type, environment of pig farm (accessibility of human feces), personal hygiene of owners, pork consumption, cooking and eating habit of pork, use of anthelmintics in pigs and owners, and knowledge on taeniasis. They were examined for testing its significance by Pearson chi-square test at $\alpha=0.05$. The data were analyzed by using SPSS (version 16).

\section{Results}

3.1. Seroprevalence of Porcine Cysticercosis in Farmed Pigs. Seroprevalence of porcine cysticercosis in farmed pigs was $15.93 \%(58 / 364)$ in the study area.

3.2. Prevalence of Households with Porcine Cysticercosis. Prevalence of households with pigs infected with T. solium cysticerci by Ab-ELISA examination was 23.15\% (47/203 households). The households with porcine cysticercosis in Pyinmana, Lewe, and Tatkon were 0/12 (0\%), 13/124 (10.48\%), and $34 / 67(50.75 \%)$, respectively.

3.3. Prevalence of Porcine Cysticercosis in Meat Inspection. The prevalence of porcine cysticercosis investigated by meat inspection was $23.67 \%$ (71/300). All the infected pigs presented parasites located in the tongue. Only in one pig, another parasite was found, located in the heart. In none of the animals evaluated, parasites were found in the other locations examined. The prevalence in slaughterhouses of Pyinmana, Lewe, and Tatkon townships was 22\% (44/200), $23.33 \%(7 / 30)$, and $28.57 \%(20 / 70)$, respectively.

3.4. Risk Factors Associated with Porcine Cysticercosis. Univariate analysis of hypothesized risk factors of gender (OR $=3.0 ; 95 \% \mathrm{CI}=1.7-5.4)$, increased age $(\mathrm{OR}=2.3 ; 95 \% \mathrm{CI}$
$=1.2-4.2)$, husbandry system $(\mathrm{OR}=5.1 ; 95 \% \mathrm{CI}=2.4-11.2)$, feed type $(\mathrm{OR}=16.9 ; 95 \% \mathrm{CI}=2.3-124.3)$, no hand washing habit before feeding $(\mathrm{OR}=31.5 ; 95 \% \mathrm{CI}=4.3-230.9)$, not using anthelmintic in pigs $(\mathrm{OR}=11.9 ; 95 \% \mathrm{CI}=5.0-28.5)$ and owner $(\mathrm{OR}=2.5 ; 95 \% \mathrm{CI}=1.4-4.4)$, and pork consumption of owner (OR $=37.4 ; 95 \% \mathrm{CI}=9.0-156.1)$ was significantly associated with Cysticercus cellulosae infection $(P<0.05)$. The distribution and odds ratio of significant risk factors concerning porcine cysticercosis are shown in Table 2.

\section{Discussion}

In Southeast Asia, pigs are an important source of food and economic important for smallholder farmers. Older pigs may be penned or tethered although common raising practice of pigs is freely roaming in the village [14]. In Myanmar, most of the pig farmers are smallholders and practice as free-range or backyard farming. In Myanmar, most of the pig farmers usually keep the weaned pigs until six to eight months of age and then send to slaughterhouse. In the village, every household keeps at least one pig not only for table waste feeding to pigs but also for extra income. Most farms are having the habit of feeding waste materials such as swill and kitchen leftover, broken rice, rice bran, groundnut meal, sesame meal and local forage, and poor sanitation.

The present study is the first report of T. solium cysticercosis in pigs in Myanmar. This investigation showed relatively high prevalence of porcine cysticercosis in the study area. Pigs in the study area positive for cysticercosis have been exposed to T. solium eggs. Among the 17 hypothesized risk factors, eight factors were evaluated as having association.

The gender of pigs (being female) was significantly associated with porcine cysticercosis in this study. It can be explained that female pigs were for kept long time for breeding purpose than male and so they have more risk to 
get exposed to T. solium eggs. However, Jayashi et al. [15] reported that gender was not a significant risk factor for porcine cysticercosis.

The present study demonstrated that the older the pigs, the greater the chance to get infection. These results are in agreement with those reported by Pouedet et al. [16], Jayashi et al. [15], Sarti et al. [17], García et al. [18], and Pondja et al. [19]. Older pigs might also have greater chance to get exposed to $T$. solium eggs than younger ones. They might have much time to develop cyst and trigger the production of circulating antibodies. Besides, it could be possible that younger pigs are protected during their first months of life against parasite infection, due to the presence of maternal cysticercus antibodies and they become susceptible later after the slow clearance of those antibodies.

The result showed that pigs from households practiced semi-intensive system (the pigs are allowed to roam freely in the environment and only panned or tethered at feeding time and night) were more likely to have porcine cysticercosis than intensive (the pigs are kept in the backyard or corral and not allowed to roam) pigs. Therefore, semi-intensive management system represented as an important risk factor for porcine cysticercosis in the study area as the pigs in this practice could access the infected human faeces. Accessibility of infected human faeces is the main source for porcine cysticercosis $[17,19,20]$.

Among the feed types used in pig farms, feeding of kitchen waste is significantly associated with cysticercosis. In the farms, most of housewives usually collect swill in poor cleanliness containers from neighboring houses. This might be contaminated with $T$. solium eggs from infected food preparers of swill collected houses. So the collected swill should be cooked thoroughly before feeding to prevent infection including cysticercosis. Human taeniasis is the main source for porcine cysticercosis [21].

Use of anthelmintic in pigs and owners was significantly associated in this study. By interviewing the farmers and township veterinary officers, the most common used anthelmintic is albendazole in human and ivermectin in pigs. Although ivermectin cannot kill any larvae of cestode, albendazole can kill these larvae. Not having taeniasis in owners is preventive factor against cysticercosis [18].

Although all the farmers wash their hands after feeding the pigs, only $21.2 \%$ famers (43/203) wash their hands before feeding. All cysticercosis positive samples were from those who do not practice hand-washing habit. Therefore, hand-washing is a crucial factor for prevention of porcine cysticercosis. However, there was no literature about this factor associated with porcine cysticercosis. But health education and sanitary infrastructure are involved in the control measure for swine cysticercosis [17].

About half of farmers (114/203) consume the pork curry in this study. All positive samples were from the owners consumed pork (51 households). They might have taeniasis and cysticercosis due to cooking habit and poor sanitation. Pork consumption of owners is also one of the risk factors in survey of porcine cysticercosis [19].

Nine hypothesized risk factors not included in analysis were breed of pigs, place of purchase, presence of latrine, hand-washing after feeding the pigs, source of water for pigs, cleanliness of water, knowledge on taeniasis and cysticercosis, and occurrence of cyst in pork. In this study, all pigs are indigenously bred. All pigs were purchased from within their township. All farmers have latrines using water, but the children do not use latrine and are used for defecation out of latrine. Some farmers washed the hands before feeding the pigs and all farmers washed their hands after feeding. All farmers used water from wells having good sanitation. All farmers did not have the knowledge on taeniasis and cysticercosis and they have never seen the cysts in the pork in the study area.

The presence of zoonotic agent, Cysticercus cellulosae, may depend on intrinsic factors: age, gender, and extrinsic factors: pig husbandry system, hand-washing habit of owner, use of kitchen waste as pig feed, not using anthelmintic in pigs and owners, and pork consumption of owner in the study area. Presence of this infection is of public health importance because it may lead to the occurrence of neurocysticercosis in human.

Although the occurrence of human neurocysticercosis has not been reported yet in Myanmar, all public should take awareness of potential risk factors due to the prevalence with high percentage observed in this study. Myanmar has no national monitoring program for $T$. solium cysticercus spp. in these animals yet. Therefore, it is advisable to monitor whether there is high or low prevalence of T. solium cysticercosis in the whole country. It could also be suggested that confinement housing system should be developed in pig industry of Myanmar to efficiently prevent porcine cysticercosis. For practicing sanitary and culinary habit, thorough cooking education programs should also be implemented for both swine breeders and consumers so as to prevent taeniasis in human and porcine cysticercosis and also other zoonotic helminth diseases in Myanmar.

This prevalence with relatively high percentage of porcine cysticercosis (15.93\%) in Ab-ELISA and $23.67 \%$ in slaughtered pigs indicates the presence of human taeniasis and it also leads to the associated risk of human cysticercosis and neurocysticercosis. Occurrence of porcine cysticercosis poses a serious problem to human health and the economy. Therefore, it is required to monitor porcine cysticercosis prevalence in the whole country.

\section{Conflict of Interests}

The authors declare that this work has not been inappropriately influenced by any financial or personal factors and they have no competing interests.

\section{Acknowledgments}

This study was partially financially supported by Nay Pyi Taw Development Committee, Nay Pyi Taw, Myanmar. The authors are grateful to Townships Veterinary Officers from Lewe and Tatkon townships and all pig owners in three townships for their good cooperation. 


\section{References}

[1] A. Carpio, A. Escobar, and A. W. Hauser, "Cysticercosis and epilepsy: a critical review," Epilepsia, vol. 39, no. 10, pp. 10251040, 1998.

[2] WHO, Neglected Tropical Diseases in the World Today, 2010.

[3] I. K. Phiri, H. Ngowi, S. Afonso et al., "The emergence of Taenia solium cysticercosis in Eastern and Southern Africa as a serious agricultural problem and public health risk," Acta Tropica, vol. 87, no. 1, pp. 13-23, 2003.

[4] A. Zoli, O. Shey-Njila, E. Assana et al., "Regional status, epidemiology and impact of Taenia solium cysticercosis in Western and Central Africa," Acta Tropica, vol. 87, no. 1, pp. 3542, 2003.

[5] A. Ito, T. Wandra, H. Yamasaki et al., "Cysticercosis/taeniasis in Asia and the Pacific," Vector-Borne and Zoonotic Diseases, vol. 4, no. 2, pp. 95-107, 2004.

[6] P. Dorny, R. Somers, T. C. T. Dang, V. K. Nguyen, and J. Vercruysse, "Cysticercosis in Cambodia, Lao PDR and Vietnam," Southeast Asian Journal of Tropical Medicine and Public Health, vol. 35, supplement 1, pp. 223-226, 2004.

[7] K. N. Prasad, S. Chawla, D. Jain et al., "Human and porcine Taenia solium infection in rural North India," Transactions of the Royal Society of Tropical Medicine and Hygiene, vol. 96, no. 5, pp. 515-516, 2002.

[8] V. Rajshekhar, "Epidemiology of Taenia solium in India and Nepal," Southeast Asian Journal of Tropical Medicine and Public Health, vol. 35, supplement 1, pp. 247-251, 2004.

[9] Y. Chen, L. Xu, and X. Zhou, "Distribution and burden of cysticercosis in China," Southeast Asian Journal of Tropical Medicine and Public Health, vol. 35, supplement 1, pp. 231-239, 2004.

[10] S. G. Simanjuntak and H. S. Widarso, "The current situation of Taenia solium taeniasis/cysticercosis in Indonesia," Southeast Asian Journal of Tropical Medicine and Public Health, vol. 35, supplement 1, pp. 240-246, 2004.

[11] Livestock Breeding and Veterinary Department (LBVD), Data from District office of Livestock Breeding and Veterinary Department, Livestock Breeding and Veterinary Department (LBVD), Nay Pyi Taw, Myanmar, 2013.

[12] M. Thrusfield, "Surveys," in Veterinary Epidemiology, pp. 178189, Butterwords, London, UK, 2nd edition, 1995.

[13] M. E. Boa, A. A. Kassuku, A. L. Willingham III, J. D. Keyyu, I. K. Phiri, and P. Nansen, "Distribution and density of cysticerci of Taenia solium by muscle groups and organs in naturally infected local finished pigs in Tanzania," Veterinary Parasitology, vol. 106, no. 2, pp. 155-164, 2002.

[14] H. Barennes, S. Sayasone, P. Odermatt et al., "A major trichinellosis outbreak suggesting a high endemicity of Trichinella infection in northern Laos," The American Journal of Tropical Medicine and Hygiene, vol. 78, no. 1, pp. 40-44, 2008.

[15] C. M. Jayashi, G. Arroyo, M. W. Lightowlers, H. H. García, S. Rodríguez, and A. E. Gonzalez, "Seroprevalence and risk factors for Taenia solium cysticercosis in rural pigs of Northern Peru," PLoS Neglected Tropical Diseases, vol. 6, no. 7, Article ID e1733, 2012.

[16] M. S. R. Pouedet, A. P. Zoli, L. Vondou et al., "Epidemiological survey of swine cysticercosis in two rural communities of WestCameroon," Veterinary Parasitology, vol. 106, no. 1, pp. 45-54, 2002.

[17] E. Sarti, P. M. Schantz, A. Plancarte et al., "Epidemiological investigation of Taenia solium taeniasis and cysticercosis in a rural village of Michoacan State, Mexico," Transactions of the Royal Society of Tropical Medicine and Hygiene, vol. 88, no. 1, pp. 49-52, 1994.

[18] H. H. García, A. E. Gonzalez, C. A. W. Evans, and R. H. Gilman, "Taenia solium cysticercosis," The Lancet, vol. 362, no. 9383, pp. 547-556, 2003.

[19] A. Pondja, L. Neves, J. Mlangwa et al., "Prevalence and risk factors of porcine cysticercosis in Angónia District, Mozambique," PLoS Neglected Tropical Diseases, vol. 4, no. 2, article e594, 2010.

[20] E. V. G. Komba, E. C. Kimbi, H. A. Ngowi et al., "Prevalence of porcine cysticercosis and associated risk factors in smallholder pig production systems in Mbeya region, southern highlands of Tanzania," Veterinary Parasitology, vol. 198, no. 3-4, pp. 284-291, 2013.

[21] A. Flisser, D. Correa, G. Avilla, and P. Marvilla, "Biology of Taenia solium, Taenia saginata and Taenia saginata asiatica," in WHO/FAO/OIE Guidelines for the Surveillance, Prevention and Control of Taeniosis/Cysticercosis, K. D. Murrell, Ed., pp. 1-8, Office International des Epizooties, Paris, France, 2005. 

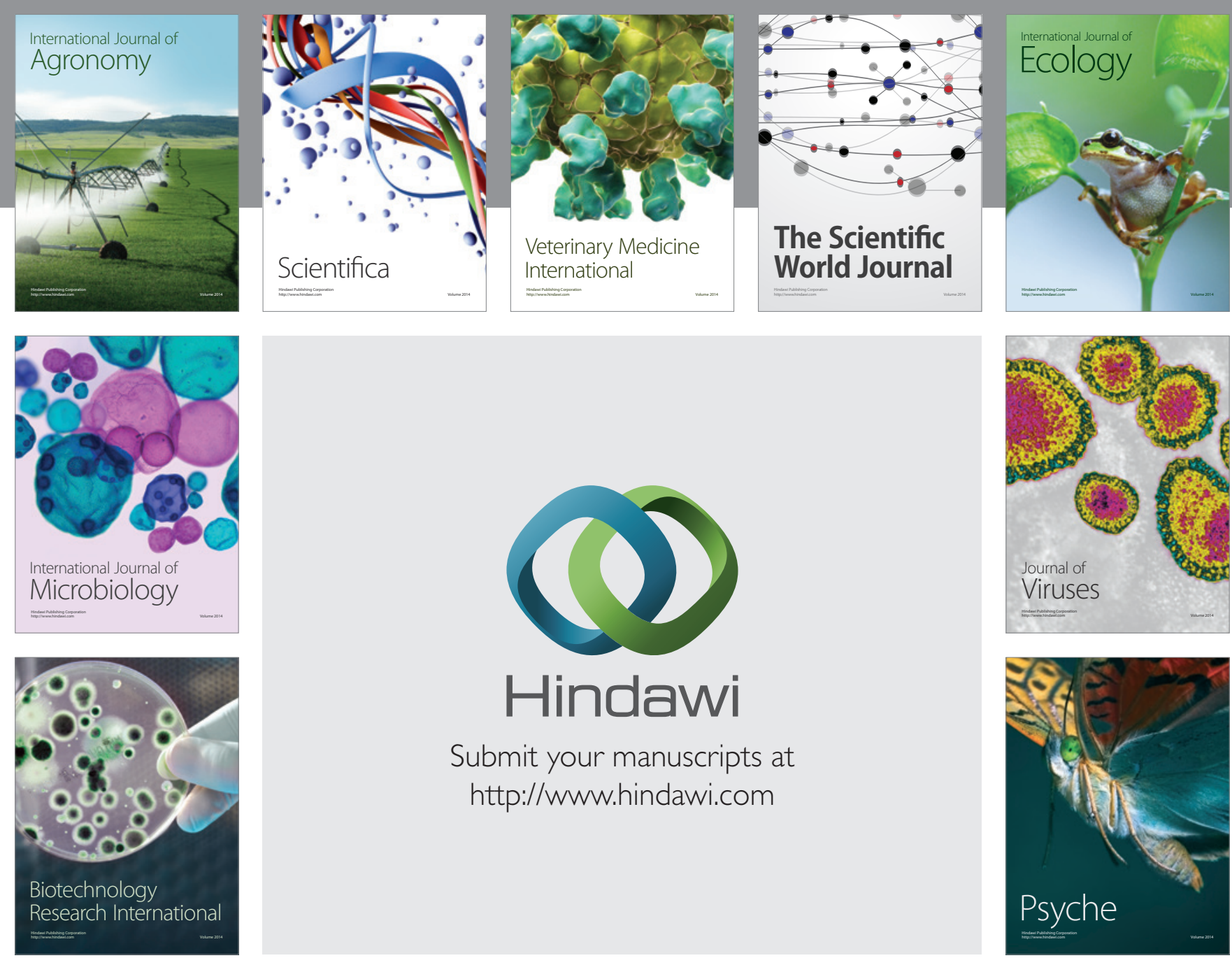

Submit your manuscripts at http://www.hindawi.com
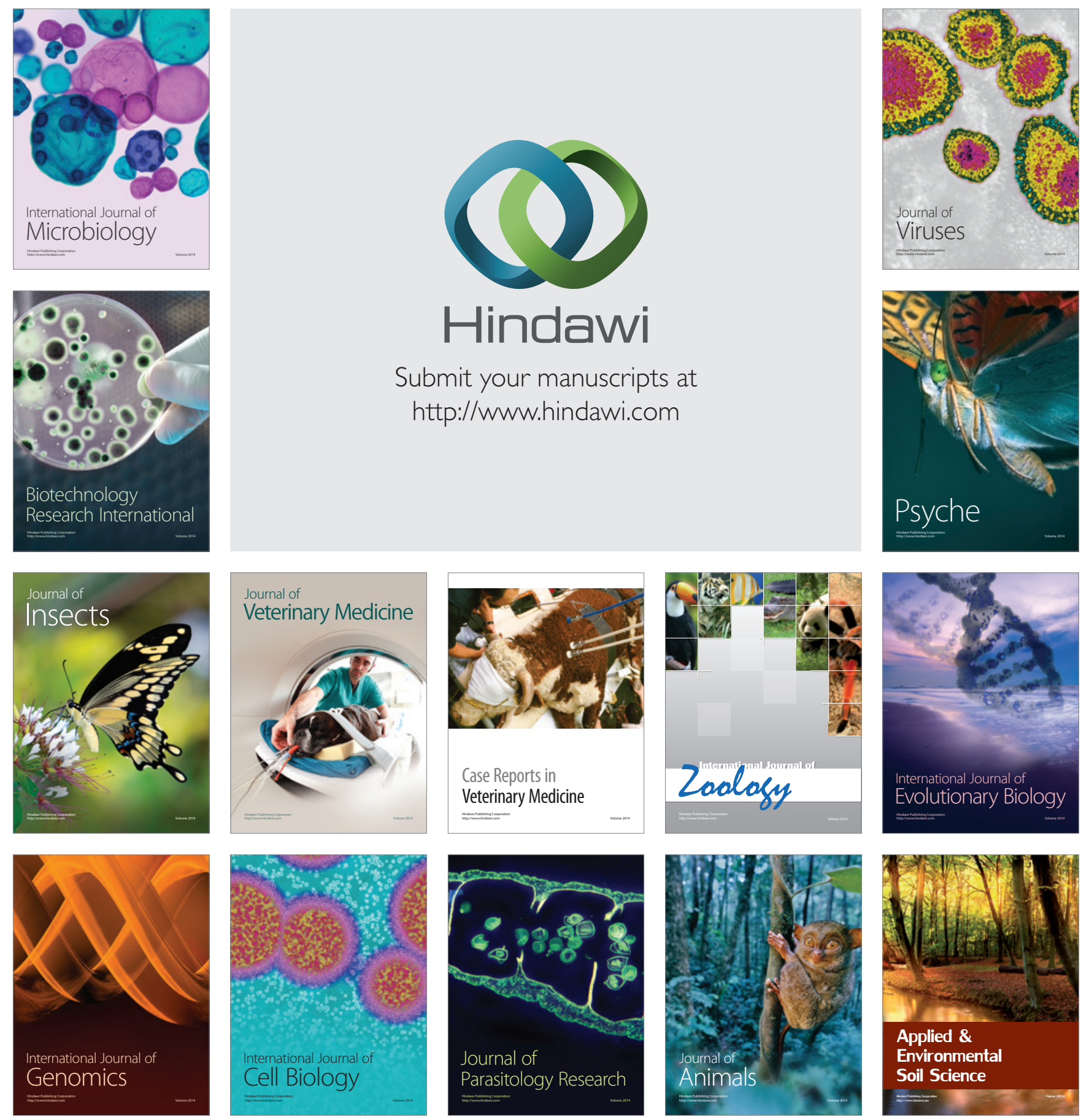